\title{
BMJ Open The SIMS trial: adjustable anchored single-incision mini-slings versus standard tension-free midurethral slings in the surgical management of female stress urinary incontinence. A study protocol for a pragmatic, multicentre, non-inferiority randomised controlled trial
}

Mohamed Abdel-Fattah, ${ }^{1}$ Graeme MacLennan, ${ }^{2}$ Mary Kilonzo, ${ }^{3}$ R Phil Assassa, ${ }^{4}$ Kirsty McCormick, ${ }^{5}$ Tracey Davidson, ${ }^{6}$ Alison McDonald, ${ }^{6}$ James N'Dow, ${ }^{8}$ Judith Wardle, ${ }^{7}$ John Norrie $^{2,8}$

To cite: Abdel-Fattah M, MacLennan G, Kilonzo M, et al. The SIMS trial: adjustable anchored single-incision mini-slings versus standard tension-free midurethral slings in the surgical management of female stress urinary incontinence. A study protocol for a pragmatic, multicentre, non-inferiority randomised controlled trial. BMJ Open 2017;7:e015111. doi:10.1136/ bmjopen-2016-015111

- Prepublication history and additional material for this paper are available online. To view these files please visit the journal online (http://dx.doi. org/10.1136/bmjopen-2016015111).

Received 8 November 2016 Revised 30 January 2017 Accepted 8 March 2017

\section{CrossMark}

For numbered affiliations see end of article.

Correspondence to Mohamed Abdel-Fattah; m.abdelfattah@abdn.ac.uk

\section{ABSTRACT}

Introduction Single-incision mini-slings (SIMS) represent the third generation of midurethral slings. They have been developed with the aim of offering a true ambulatory procedure for treatment of female stress urinary incontinence (SUI) with reduced morbidity and earlier recovery while maintaining similar efficacy to standard midurethral slings (SMUS). The aim of this study is to determine the clinical and cost-effectiveness of adjustable anchored SIMS compared with tension-free SMUS in the surgical management of female SUl, with 3-year follow-up. Methods and analysis A pragmatic, multicentre, noninferiority randomised controlled trial.

Primary outcome measure The primary outcome measure is the patient-reported success rate measured by the Patient Global Impression of Improvement at 12 months. The primary economic outcome will be incremental cost per quality-adjusted life year gained at 12 months.

Secondary outcome measures The secondary outcomes measures include adverse events, objective success rates, impact on other lower urinary tract symptoms, healthrelated quality of life profile and sexual function, and reoperation rates for SUI. Secondary economic outcomes include National Health Service and patient primary and secondary care resource use and costs, incremental costeffectiveness and incremental net benefit.

Statistical analysis The statistical analysis of the primary outcome will be by intention-to-treat and also a perprotocol analysis. Results will be displayed as estimates and $95 \% \mathrm{Cls}$. Cls around observed differences will then be compared with the prespecified non-inferiority margin. Secondary outcomes will be analysed similarly. Ethics and dissemination The North of Scotland Research Ethics Committee has approved this study
(13/NS/0143). The dissemination plans include HTA monograph, presentation at international scientific meetings and publications in high-impact, open-access journals. The results will be included in the updates of the National Institute for Health and Care Excellence and the European Association of Urology guidelines; these two specific guidelines directly influence practice in the UK and worldwide specialists, respectively. In addition, plain English-language summary of the main findings/results will be presented for relevant patient organisations.

Trial registration number ISRCTN93264234. The SIMS study is currently recruiting in 20 UK research centres. The first patient was randomised on 4 February 2014, with follow-up to be completed at the end of February 2020.

\section{Background}

Urinary incontinence (UI) is a common and potentially debilitating condition among women. ${ }^{1}$ In the UK, the estimation for clinically significant UI symptoms (age $>40$ years) was 6 million women $(40 \%)$, with 1 million women $(6.2 \%)$ reporting to be significantly bothered by their symptoms. UI was socially disabling in 0.33 million $(2.2 \%) .^{2}$ UI has a negative impact on a woman's social, physical and psychological well-being, leading to embarrassment, low self-esteem and social isolation. UI is associated with negative effects on the productivity of working women, with some avoiding employment because of fear of embarrassing situations. ${ }^{3}$ UI has significant cost implications to the individual and the 
health service. The total annual cost to the UK National Health Service (NHS) for the management of women over the age of 40 with UI was $£ 301$ million or $0.3 \%$ of the NHS budget. ${ }^{4}$ Costs borne by women in terms of out-ofpocket expenses were $£ 230$ million $^{5}$ or $£ 290$ per woman per year. ${ }^{6}$ All values reported are inflated to 2009 values.

Stress urinary incontinence (SUI) is the most common type of UI in premenopausal women, accounting for almost $50 \%$ of cases. ${ }^{7}$ It is defined as involuntary leakage of urine on effort, or exertion, or on sneezing or coughing. Initial management of SUI includes conservative therapy such as pelvic floor muscle training (PFMT), biofeedback, electrical stimulation or drugs. When conservative therapy fails, in about one-third of cases, surgery is the next option ${ }^{7}$ Of the surgical treatments available, tension-free standard midurethral slings (SMUS; retropubic tension-free vaginal tape (RP-TVT) and transobturator tension-free vaginal tape (TO-TVT)) are the most commonly performed procedures for SUI, resulting in 11000 finished consultant episodes in England in 2009-2010, with estimated costs of £2044/ procedure, ${ }^{8}$ that is, a total of $£ 22.5$ million/year. The Cochrane review of minimally invasive midurethral slings (MUS $)^{9}$ concluded that there was no evidence of significant differences in patient-reported outcomes between RP-TVT and TO-TVT, and therefore the control arm for the proposed randomised controlled trial (RCT) is a pragmatic combination of these two types of SMUS. Analysis of British Society of Urogynaecology database showed that the vast majority of SMUS in the UK are done under general anaesthesia (GA) or deep intravenous patient sedation. ${ }^{10}$

Single-incision mini-slings (SIMS) represent the third generation of MUS; they have been developed with the aim to offer a true ambulatory procedure for treatment of SUI with reduced morbidity and earlier recovery while maintaining similar efficacy to SMUS. The National Institute for Health and Care Excellence (NICE) undertook an interventional procedure overview of SIMS ${ }^{1}$ for the management of SUI in women in July 2007 (NICE guidance/IP398): there was no RCT evidence and only small case series data were available. The report concluded that the current evidence on the safety and efficacy of SIMS was inadequate in quality and quantity, and recommended that SIMS should only be performed in the context of research. Similarly, the Cochrane review of minimally invasive MUS found no randomised evidence evaluating SIMS. ${ }^{9}$

\section{Rationale for the study}

The European guidelines ${ }^{11}$ on the management of urinary incontinence describe two concepts of MUS for the surgical treatment of SUI in women: (1) Tension-free MUS that include all MUS that depend on their post-insertion fixation mechanism on friction to nearby tissues within their relatively long trajectory of insertion such as SMUS (both RP-TVT and TO-TVT); one type of non-anchored SIMS (Contasure-Needleless) also fits into this group. (2) Anchored MUS that include all other SIMS and other anchored slings such as Remeex TRT; the latter is mainly used in women with recurrent SUI. ${ }^{12}{ }^{13}$ SIMS fundamentally differs from SMUS because they have a shorter trajectory of insertion and therefore need a robust anchoring mechanism to the obturator complex with a strong post-insertion pull-out force. All currently available SIMS share the same tape material (type 1 polypropylene) and the insertion technique through a single vaginal incision; however, they differ in the type/robustness of the anchorage mechanism used. ${ }^{14}{ }^{15}$ A number of recently developed SIMS, such as Ajust, Altis, and TFS, have an added advantage that allow post-anchorage adjustment of the sling tension and have been shown in independent animal studies, assessing their immediate and delayed extraction forces, to be associated with the strongest and most robust anchoring mechanism to the obturator complex. ${ }^{1415}$

A multicentre prospective cohort study of adjustable anchored SIMS-Ajust in 100 women has shown its acceptability $(75 \%)$ and feasibility (97\%) to be done under local anaesthesia (LA). ${ }^{16}$ We recently concluded our multicentre prospective pilot $\mathrm{RCT}^{17}$ where 137 women were randomised to adjustable anchored SIMS-Ajust $(n=69)$, performed under LA, versus SMUS (TVT-O; $\mathrm{n}=68$ ). At a minimum of 12 months' follow-up, there were no significant differences in the patient-reported success rate (OR $0.895 ; 95 \%$ CI 0.344 to $2.330 ; \mathrm{p}=1.000$ ), objectives success rate (OR $0.929 ; 95 \%$ CI 0.382 to $2.258 ; \mathrm{p}=1.00$ ) and reoperation rates (OR $0.591 ; 95 \%$ CI 0.136 to 2.576 ; $\mathrm{p}=0.721$ ) between both groups. Comparable number of women reported significant improvement in their quality of life (QoL) ( $\mathrm{p}=0.190)$ and sexual function $(\mathrm{p}=0.699)$ in both groups. Similar results were recently reached by a Deutsch group in a similar small RCT. Similar results were reported in observational studies with varying cohort sizes: patient-reported and objective success rates of $85 \%-91 \%$ up to 12 -month follow-up. ${ }^{18-21}$

A recent updated systematic review and meta-analysis ${ }^{22}$ compared the effectiveness and complications of SIMS versus SMUS for the surgical management of female SUI. The authors considered TVT-Secur to be clinically irrelevant, having been excluded from clinical practice and excluded the relevant RCTs, hence included a total of 26 RCTs ( $\mathrm{n}=3308$ women). Meta-analyses showed that at a mean follow-up of 18 months, there was no evidence of significant differences between SIMS and SMUS in patient-reported and objective cure rates (relative risk (RR): $0.94 ; 95 \%$ CI 0.88 to 1.00 ; and RR: $0.98 ; 95 \%$ CI 0.94 to 1.01 , respectively). Interestingly, SIMS had significantly lower postoperative pain scores, earlier return to normal activities and earlier return to work (weighted mean difference (WMD): $-2.94 ; 95 \%$ CI -4.16 to -1.73 ; WMD: -5.08 ; $95 \% \mathrm{CI}-9.59$ to -0.56 ; and WMD: $-7.20 ; 95 \% \mathrm{CI}-12.43$ to -1.98 , respectively). The authors reported that SIMS had a trend towards higher rates of repeat continence surgery, although it did not reach statistical significance (RR: $2.00 ; 95 \%$ CI 0.93 to 4.31 ). An exploratory subgroup 
analysis evaluating adjustable anchored SIMS (Ajust and TFS) versus TO-TVT included four RCTs and showed no evidence of significant differences in patient-reported or objective cure rates. However, it is important to note that they found no RCTs evaluating Altis. ${ }^{22}$ The authors concluded that "on excluding TVT-Secur, there was no evidence of significant differences in patient-reported and objective cure between currently used SIMS and SMUS at midterm follow-up while associated with more favourable recovery time, ${ }^{22}$ The authors urged caution in the interpretation of results due to the heterogeneity of the small trials included, lack of blinding of the assessors which can be a source of bias, the level of incomplete data leading to attrition bias and the relatively short term of follow-up.

Evidence of longer term outcomes for adjustable anchored SIMS are now emerging. In July 2012, Sivaslioglu et $a l^{23}$ reported a 5-year follow-up for their RCT of TFS versus SMUS $(n=80)$. The results showed no evidence of significant difference in objective and patient-reported success rates (TFS $83 \%-89 \%$ vs SMUS $75 \%-78 \%$, respectively). Similarly, Naumann et $a l^{24}$ reported a 2-year follow-up of observational study of 51 women who underwent SIMS-Ajust; the patient-reported success rate was $86 \%$.

The cost-effectiveness of any new technology is a prerequisite for its adoption in clinical practice. Our team performed the first health economic analysis of adjustable anchored SIMS (Ajust) compared with SMUS (TVT-O). ${ }^{25}$ The health economic outcome measures were incremental costs to the health services, patient quality-adjusted life year (QALYs) and incremental cost per QALY. Results have shown an incremental total cost savings to the health service of $£ 142 /$ procedure with adjustable anchored SIMS, not counting the further potential economic gain of earlier return to work in these women. There were no significant differences in QALYs generated compared with SMUS: 95\% CI -0.008 to 0.002 . Assuming these results were generalisable to all currently performed MUS procedures in England and Wales (approximately 11000 in 2010), ${ }^{10}$ our analyses suggest the potential for substantial cost savings to the NHS in the UK of about $£ 1.5$ million per year. However, these results have to be confirmed in the definitive RCT.

The above evidence has led to a consensus among urologists and urogynaecologists that an adequately powered RCT with clinical effectiveness as the primary end point is now timely and required to inform surgeons, patients and decision makers with the most clinically effective, cost-effective surgical treatment for primary SUI, which is associated with the least burden on patients' QoL and NHS resources.

\section{Study objectives}

The aim of this pragmatic multicentre RCT is to determine the clinical effectiveness and cost-effectiveness of adjustable anchored SIMS compared with tension-free SMUS in the surgical management of female SUI.
The hypothesis being tested is that patient-reported success rate following surgical treatment with adjustable anchored SIMS procedures is non-inferior to tension-free SMUS, while the former is associated with less postoperative pain, shorter hospital stay, earlier recovery and consequently earlier return to usual activities/work, and is more cost-effective than SMUS.

The primary objective is to compare SUI outcomes in terms of patient-reported success rates as measured by the Patient Global Impression of Improvement (PGI-I) at 12 months.

The primary economic objective is to compare cost-effectiveness measured in terms of QALYs derived from responses to the EQ-5D and the ICIQ-LUTSqol over the follow-up period.

The secondary objectives are to compare objective success rates (24-hour pad test/home cough stress test) and other patient-reported outcomes, including postoperative pain scores and health-related QoL using the ICIQ-LUTSqol, impact on other urinary symptoms (International Consultation on Incontinence Questionnaire -Female Lower Urinary Tract Symptoms (ICIQ-FLUTS) ), impact on sexual function (ICIQ-FLUT-Sex/PISQ-IR), complication rates, disease recurrence and costs to the NHS and patients.

\section{METHODS \\ Study design}

This is a pragmatic, multicentre, non-inferiority RCT comparing adjustable anchored SIMS with tension-free SMUS in surgical management of SUI in women. The trial structure is presented below (figure 1).

\section{Intervention to be evaluated}

The interventions being compared are (1) tension-free SMUS, including RP-TVT and TO-TVT, and (2) adjustable anchored SIMS, which fulfil the following criteria of robust anchorage and postinsertion adjustability:

- SIMS is made of type 1 polypropylene mesh: monofilament and macroporous (pore size $=75 \mu \mathrm{m}$ )

- robustly anchored to obturator complex (robust insertion is defined as immediate pullout force $=12 \mathrm{New}$ tons $(\mathrm{N})$ and/or 4 weeks' pullout force $=30 \mathrm{~N}$ )

- fully adjustable sling postinsertion

- proven feasibility to be done under LA

- minimum of level 2 evidence showing their safety and short-term (minimum 3 months) patient-reported outcomes.

SMUS will be performed under GA or deep intravenous sedation, while adjustable anchored SIMS will be done under LA as an opt-out policy (ie, LA will be the standard type of anaesthesia for adjustable anchored SIMS unless specifically declined by a participant requesting GA). Furthermore, participant's requests for conversion to GA will be respected at any stage of the procedure. A standard LA protocol, which we have previously published and 
successfully used in two previous studies,${ }^{16}{ }^{17}$ will be used as a guidance (online supplementary appendix 1).

All participants, in both arms, will receive preoperative analgesia (30-60 min prior to the operation): paracetamol and non-steroidal anti-inflammatory drug (diclofenac sodium or ibuprofen); a vaginal application of EMLA cream (a 5\% emulsion preparation, containing $2.5 \%$ each of lidocaine/prilocaine); and optional $10 \mathrm{~mL}$ of intraurethral Instillagel (anaesthetic, antiseptic lubricant). All participants would receive preoperative/ intraoperative prophylactic broad spectrum antibiotics. A cystoscopy (rigid or flexible) will be performed in all women following insertion of the sling, regardless of the study arm. It is worth noting that rigid cystoscopy was well tolerated by all women under LA in the pilot RCT. No vaginal packs or catheters would be routinely inserted.

\begin{tabular}{|l|}
\hline Ineligible (all exclusion criteria) \\
- Anterior or Apical Prolapse $\geq$ POP- \\
Q Stage 2 (POP-Q = pelvic organ \\
prolapse quantification score) \\
- Previous incontinence surgery (for \\
SUI or overactive bladder \\
Symptoms - OAB) \\
- Mixed incontinence with pre- \\
dominant OAB symptoms that \\
failed to be controlled on \\
conservative treatment (Bladder \\
retraining, PFMT and/or anti- \\
muscarinic treatment) \\
- Neurological conditions e.g. MS, \\
spinal cord injuries \\
- Concomitant surgery at time of SUI \\
surgery \\
- Previous Pelvic irradiation \\
- Pregnancy or planning for a family \\
- Inability to understand the \\
information leaflet and consent \\
form in English. \\
\hline
\end{tabular}

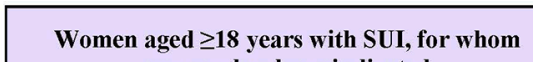
surgery has been indicated

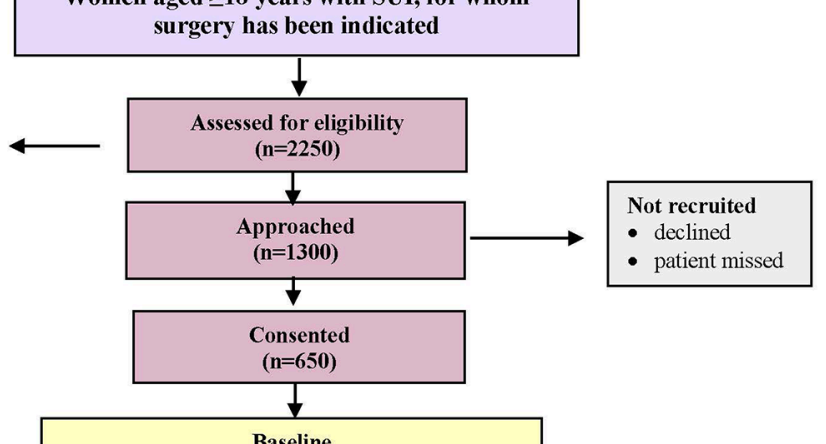

EQ-5D, ICIQ-LUTSqol, ICIQ-FLUTS, ICIQ-SF \& UPS, ICIQ-FLUT-Sex or PISQ-IR $24 \mathrm{hr}$ pad test. Home Continence Stress Test
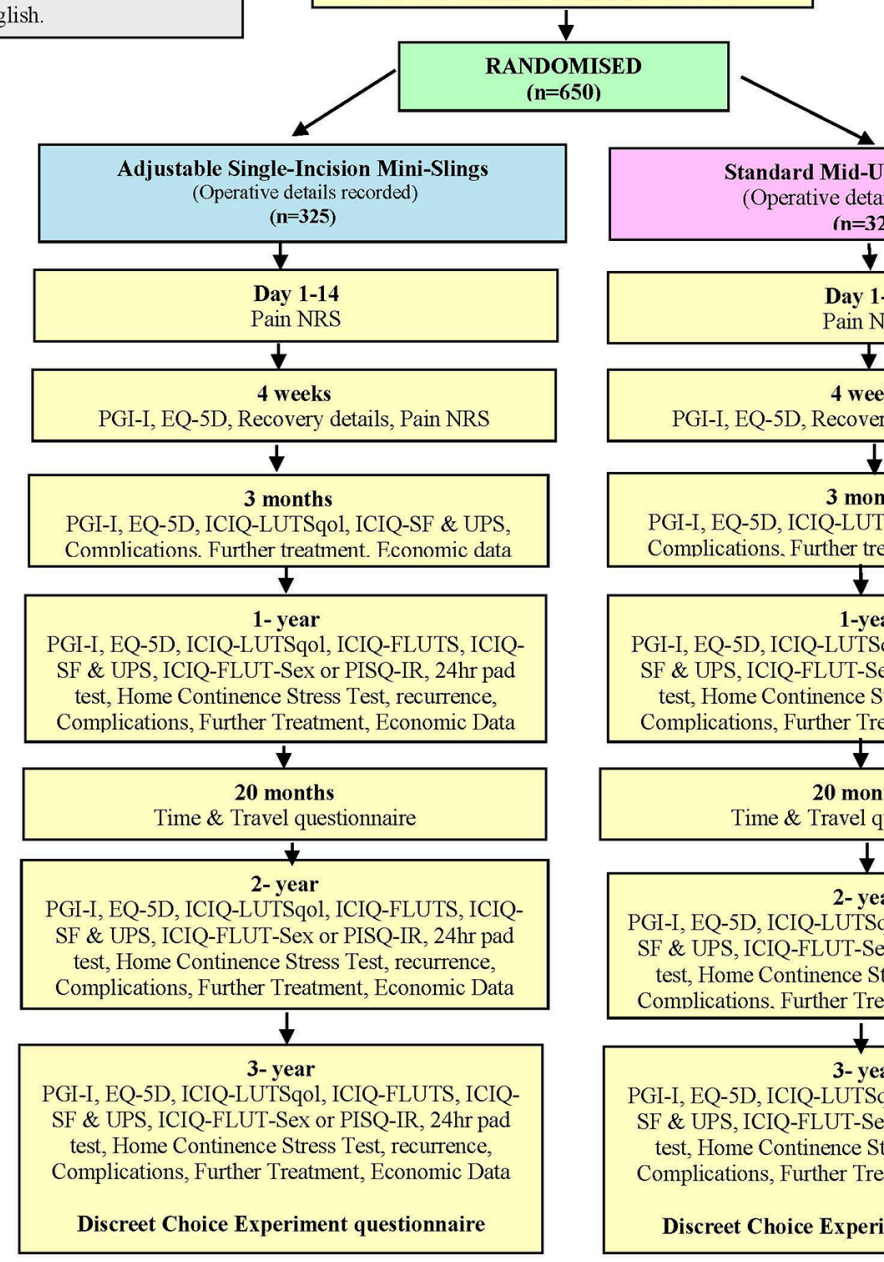

Figure 1 SIMS study flow chart. ICIQ-FLUTS, International Consultation on Incontinence Questionnaire - Female Lower Urinary Tract Symptoms; NRS, Numerical Rating Scale; PFMT, pelvic floor muscle training; SIMS, single-incision mini-slings; SUI, stress urinary incontinence; UPS, Urgency Perception Scale. 
Postoperatively all participants will undergo voiding assessment, including assessment for postvoiding residual urine volume (PVR) using a bedside bladder scanner (online supplementary appendix 2, guidance protocol and flow chart for postoperative voiding assessment).

\section{Adjustable anchored SIMS}

A standard combination of fast and delayed action LA (dose-dependent on participant's body weight) will be infiltrated vaginally into either side of the urethra, the vaginal angles (sulci) and behind the inferior pubic ramus into the obturator complex (eg, using a curved black spinal needle and/or pudendal block needle). Women will be accompanied by a nurse for support. All participating surgeons will use an adjustable anchored SIMS that meets the prespecified criteria described below. A standardised insertion technique will be used by all surgeons following the original description of the particular SIMS used. Most adjustable anchored SIMS, however, have a fairly similar procedure of insertion. We describe below the standard insertion steps for the adjustable anchored SIMS (Ajust-CR Bard and Altis-Coloplast): women will be positioned in lithotomy position with hips flexed at $90^{\circ}-100^{\circ}$. LA infiltration is as above; a suburethral vertical vaginal incision $(\sim 1.5 \mathrm{~cm})$ will be made; and bilateral paraurethral tunnels will be created reaching to the posterior margin of the inferior pubic ramus but without piercing the obturator membrane. Further infiltration of LA into the obturator membrane will be done, and SIMS, with the 'fixed anchor' end mounted on the applicator, would be introduced through the predissected paraurethral tunnel until reaching behind the inferior pubic ramus. The applicator would then pivot slowly behind the ramus and through the obturator complex allowing the fixed anchor to maintain its position in the obturator membrane and muscles at points equivalent to 10 and 2 o'clock in relation to the urethral orifice. The insertion steps would be repeated on to the other side allowing the 'adjustable anchor' to be fixed in the contralateral obturator complex. The SIMS is now robustly anchored and the tension would then be adjusted as required to achieve continence while avoiding voiding difficulty. Performing the cough stress test can prove very helpful in this adjustment process and is recommended. The adjustable anchor would then be locked in the case of the Ajust (not required with Altis), and a cystoscopy will be performed to exclude perforation and the vaginal incision closed.

\section{Tension-free SMUS}

The choice of SMUS whether retropubic or transobturator will depend on the surgeon's experience. We expect a $50 \%$ representation of each type of SMUS in the control arm.

\section{Retropubic tension-free vaginal tape}

RP-TVT will be type 1 polypropylene mesh (monofilament and macroporous: pore size $\geq 75 \mu \mathrm{m}$ ). The tension-free vaginal tape (TVT) procedure was developed by Ulmsten et $a .^{26}$ The procedure will be done under GA or intravenous sedation as per the standard practice of each surgeon. The bladder will be emptied with a Foley catheter. Close to the superior rim of the pubic bone, two $1 \mathrm{~cm}$ long transverse incisions $3 \mathrm{~cm}$ either side of the midline will be made after injection of LA into the abdominal skin just above the symphysis pubis, down along the back of the pubic bone to the retropubic space and vaginally into the periurethral area. An incision $\sim 1.5 \mathrm{~cm}$ long will be made in the midline of the suburethral vaginal wall, followed by dissection of the periurethral tunnels to allow introduction of the TVT needle. A stent will be inserted into the Foley catheter to deviate the urethravesical junction away from the path of the needle. The TVT needle perforates the urogenital diaphragm and will be brought up to the abdominal incision 'shaving' the back of the pubic bone. The procedure will then be repeated on the other side, and a cystoscopy will be performed to exclude perforation. The cough stress test may then be performed, according to the surgeon's standard technique, and the sling adjusted in a tension-free fashion and the incisions are closed.

\section{Transobturator tension-free vaginal tape}

TO-TVT will be type 1 polypropylene mesh (monofilament and macroporous: pore size $\geq 75 \mu \mathrm{m}$ ). All procedures will be performed under GA as originally described by Delrome ${ }^{27}$ and de Leval ${ }^{28}$ for the outside-in and inside-out routes, respectively. Women are positioned in lithotomy position with hips flexed at $100^{\circ}-110^{\circ}$, and LA may be infiltrated into the vaginal angles; the latter is not a standard practice, however is recommended in a similar regimen to the one used in the adjustable SIMS insertion (above). A $\sim 1.5 \mathrm{~cm}$ suburethral longitudinal vaginal incision will be made and bilateral paraurethral tunnels created reaching to the posterior margin of the inferior pubic ramus. Bilateral groin incisions are made $1-2 \mathrm{~cm}$ lateral to the labiofemoral fold and $2 \mathrm{~cm}$ above the level of urethra. The transobturator trocar is inserted from groin incisions at $90^{\circ}$ to pierce the obturator muscles and membranes and then guided by the surgeon's finger to the vaginal incision. TO-TVT is then mounted on the trocar and the trocar is withdrawn in reverse order. The previous two steps are repeated on the contralateral side achieving TO-TVT suburethral placement, and the TO-TVT is then adjusted tension-free. For the inside-out technique of insertion, TO-TVT would be introduced in the reverse route from the vaginal incision towards the groin using the winged guide to protect the lower urinary tract (LUT). A cystoscopy will be performed to exclude LUT injury. Vaginal and skin incisions will then be closed.

\section{Study population}

The study included women aged 18 years or over with SUI who have been referred to the collaborating surgical gynaecology, urology and urogynaecology units from across the UK for treatment of SUI for whom surgery has been indicated. 


\section{Setting}

The study will be done in secondary and tertiary care acute hospital settings across the UK. NHS Grampian will be the clinical coordinating centre and house the chief investigator.

Each unit will have at least one participating surgeon who is competent in performing SIMS under LA prior to enrolling in the RCT. Ideally, the surgeon will have performed 20 adjustable anchored SIMS procedures (with 10 performed under LA), within prospective audit and results submitted to a national surgical database. The chief investigator, or a delegated expert in SIMS, will provide training in SIMS under LA for enrolling surgeons as necessary and will ensure adequate expertise of surgeons in both arms. Surgeons will be experienced in at least one type of SMUS (RP-TVT or TO-TVT) and will have performed an adequate workload in the preceding 2 years.

\section{Selection of participants}

Clinicians assess patients likely to require surgery for SUI as per standard clinical practice in their unit and as per national guidelines. Each unit will keep a $\log$ with brief details of all potentially eligible patients assessed in order to document the reasons for non-inclusion in the study (eg, reason they were ineligible, or declined to participate). This information will inform the trial CONSORT diagram.

\section{Planned inclusion and exclusion criteria Inclusion criteria}

Inclusion criteria include women $\geq 18$ years with urodynamic stress incontinence/or urodynamic mixed UI with predominant SUI bothering symptoms, in whom surgery to treat SUI has been decided by the patient and her treating clinician. Women will have failed or declined conservative treatment (including supervised PFMT) and would have completed their families. In line with the NICE guidelines, the small group of women with pure SUI and no symptoms of overactive bladder (OAB) or voiding dysfunction (VD) can be included in the study without urodynamic investigations.

Preoperative urodynamic investigations include free uroflowmetry, PVR urine volume assessment and subtracted filling cystometry. Other tests such as urethral pressure profile and leak point pressure pressures are not mandatory, however are welcome as they will inform a number of the preplanned secondary outcomes.

\section{Exclusion criteria}

Women will be excluded if they have one or more of the following:

- anterior or apical prolapse $\geq$ POP-Q stage 2 (POP-Q, Pelvic Organ Prolapse Quantification score)

- previous surgery for SUI

- mixed incontinence with predominant OAB symptoms (defined as OAB failed to be controlled on con- servative treatment, such as bladder retraining, PFMT and/or antimuscarinic treatment)

- neurological conditions, for example, multiple sclerosis (MS), spinal cord injuries

- concomitant surgery at time of SUI surgery

- previous pelvic irradiation

- pregnancy or planning for a family

- inability to understand the information leaflet and consent form in English.

\section{Recruitment and study procedures}

Identifying participants

Local procedures at the participating hospitals are different; hence, the timing and patients' approach and the consent process will vary accordingly with special emphasis to meet individual patient's needs. The patient information sheet will be sent to patients together with their clinic appointments where possible, ensuring that they have ample time ( $>24$ hours) for consideration before being approached by the research team at the clinic.

Patients likely to require surgery for SUI and who meet the eligibility criteria will be identified at the preassessment clinics, urodynamic clinics and outpatient urology/gynaecology clinics by the consultant, research nurse $(\mathrm{RN})$ or a designated team member. The consultant/RN will introduce the study to the patient and provide her with the patient information sheet as appropriate, answer any queries, and if appropriate the participant may sign the consent form, receive the baseline assessment pack for completion at home and bring back on the day of surgery or send back to the site using prepaid post.

Patients whose first approach is at the clinic will be given as much time as they require to consider participation; patients may make a decision to participate at this time or may agree to be contacted at home by the local $\mathrm{RN}$. If a patient agrees to be contacted at home, she will receive a telephone call from the local $\mathrm{RN}$ to discuss any queries. If a patient agrees to the study at that stage, then arrangements will be made for baseline assessment and consenting; this could be done as a separate appointment or at a preadmission clinic. As above, participants can complete the baseline assessment pack at home and bring back on the day of surgery or send back to the site using prepaid post. These arrangements can be individualised for each centre.

\section{Informed consent}

The patient information leaflet explains that the trial is investigating the use of either adjustable SIMS or tensionfree SMUS for the surgical management of SUI in women. All participants will sign informed consent forms. Participants who cannot give informed consent (eg, due to incapacity) will not be eligible for participation. Pending their permission, the participants' permission, the general practitioner (GP) will be informed of their participation. 
Randomisation and allocation

Eligible and consenting participants will be randomised to one of the two study groups in a 1:1 allocation ratio using the randomisation application at the trial office at the Centre for Healthcare Randomised Trials (CHaRT). This randomisation application will be available 24 hours a day, 7 days a week as both an interactive voice response telephone system and as an internet-based application. The randomisation will use a minimisation algorithm based on centre and previously supervised PFMT within the last 2 years (PFMT: yes/no). Women will be further randomised to receive short versus detailed sexual function questionnaire.

\section{Follow-up procedures}

Eligible patients who have given signed informed consent to participate in the study will be randomised to either SIMS or SMUS. They will be asked, at baseline, to complete the preoperative questionnaire pack, which includes few questions on participants' demographic details and preoperative health/medications. It also includes validated questionnaires for symptom severity of UI and its impact on QoL and sexual function: the EQ-5D, ICIQSF, Urgency Perception Scale (UPS), ICIQ-LUTSqol, ICIQ-FLUTS and ICIQ-FLUTS-Sex (or PISQ-IR), and to perform 24-hour pad test and home continence stress test (see online supplementary appendix 3, objective assessment of urinary incontinence within the SIMS trial - protocol).

On days 1-14 they will be asked to complete the pain score and use of analgesics questions by self-completed postoperative diary. At 4 weeks postoperatively, participants will be asked to complete a short questionnaire (on the last section of the diary) to capture pain, use of analgesia, complications, return to work/normal activities, PGI-I and EQ-5D. At 3 months postoperatively, participants will be asked to complete a number of questionnaires - to measure the PGI-I, EQ-5D, ICIQ-SF, UPS and questions related to health services resource - and to report any complications or further treatment received for UI. In addition, at 12, 24 and 36 months postrandomisation, participants will be asked to complete a questionnaire to measure the PGI-I, recurrence, further treatment received and questions related to health services resource use, in addition to all baseline assessment pack. Taking into account the inevitable waiting time between randomisation and receiving the surgical treatment (average surgical waiting list is 8-12 weeks), and in addition the clinical importance of assessing the outcomes at 12 months postoperatively, we aim to send the 1-year follow-up pack at 15 months postrandomisation. This strategy will ensure that the vast majority of participants are at least 12 months postoperative at time of capturing the primary outcome. In addition, at 20 months participants will be asked to fill out an additional economic data questionnaire, which will include the patient time and travel costs questionnaire. Sending this questionnaire at 20 months will minimise patient burden when completing the annual questionnaire. The discrete choice experiment (DCE) will be completed at the end of the 3-year follow-up period.

Questionnaires and up to two reminders will be sent to participants by post. Non-responders to the 12-month postrandomisation questionnaire will be contacted by phone for a short interview to capture the primary outcome (PGI-I; a single item question to mark the outcome of the operation as described in section 5.1). If the participant indicates at this phone call her wish to withdraw from the study, a 'Change of Status Form' will be completed as below. Participants will be sent a voucher (of modest value) as a token of appreciation for completion and return of the 3-month and follow-up questionnaires.

\section{Change of status/withdrawal procedures}

Participants will remain on the trial unless they choose to withdraw consent or if they are unable to continue for a clinical reason. If a participant withdraws consent, participant questionnaires will not be collected. A member of the research team will contact the participant by phone and complete the 'change of status form', which includes the participant's instructions on what parts, or whole, of the study they may wish to withdraw from. Unless a participant specifically declines, the research team will continue to collect relevant data from their healthcare records such as ONS and NHS central registries. Except for formal withdrawal of consent, all other changes in status will mean the participant is still followed up for all study outcomes wherever possible.

\section{Subsequent arrangements (if applicable) Informing key people}

Following formal trial entry, the Study Office will inform the participant's GP via a letter detailing information about the SIMS study and the Study Office contact details.

The local RN will file the hospital copy of the consent form in the hospital notes along with information about SIMS, give one copy to the patient, file one copy to the local site file and send one copy to the Study Office in Aberdeen.

1. use the SIMS internet database to enter data regarding the participant, including data required to complete randomisation

2. data entry onto the study database as soon as practical

3. forward a copy of study documentation when and as requested by the Study Office in Aberdeen to facilitate quality control.

\section{Notification of/by GPs}

GPs are asked to contact the Study Office if one of the participants moves, becomes too ill to continue or dies, or any other notifiable event or possible serious adverse event occurs. Alternatively, staff at the Study Office may contact the GP. 


\section{SAFETY}

The SIMS trial involves procedures for the surgical management of SUI in women which are well established in clinical practice. However adverse effects may occur during or after any type of surgery.

\section{Definitions}

An adverse event (AE) is any untoward medical event affecting a clinical trial participant. Each initial AE will be considered for severity, causality or expectedness, and may be reclassified as a serious event.

AEs are not:

- continuous and persistent disease or symptom, present before the trial, which fails to improve, such as urgency, urgency incontinence, $\mathrm{VD}$, pain or dyspareunia

- treatment failure: persistence or recurrence of UI

- worsening pain or where the site of pain changes is an AE.

A serious adverse event ( $\mathrm{SAE}$ ) is any $\mathrm{AE}$ that:

- results in death

- is life-threatening (ie, the participant was at risk of death at the time of the event; it does not refer to an event that hypothetically might have caused death if it was more severe)

- results in persistent or significant disability or incapacity

- requires an unplanned readmission to the hospital (defined as 'participant admitted as an in-patient with $\geq 1$ night hospital stay'); this excludes hospital ward attenders for minor issues such as lower urinary tract infection, voiding difficulties or other issues considered by the PI to be minor; this information will be routinely collected on the postoperative form and/or the supplementary hospital visit form as appropriate

- requires prolongation of existing hospitalisation (defined as $>36$ hours postoperative hospital stay); this excludes prolongation of hospital stay for minor issues such as voiding difficulties; such information will be routinely collected on the operation and clinical data form; prolongation of hospital stay due to social/geographical reasons will not be considered

- is otherwise considered medically significant by the investigator.

Note: Hospitalisations for treatment planned prior to randomisation and hospitalisation for elective treatment of a pre-existing condition, or complication arising from either, will not be considered as an (S) AE.

\section{Specific expected AEs}

In this surgical trial the following events are potentially expected:

- Intraoperative complications: bleeding, bladder/urethral injury, bowel injury, nerve injury (obturator/ dorsal nerve of clitoris), injury to blood vessels, hypersensitivity to the local/general anaesthetics and/or any of the medications or materials used, pain, shak- ing/dizziness, change of procedure or device and/or type of anaesthesia

- Immediate postoperative complications: pain in the hip/ thigh or the vagina, infection (chest, urinary tract), bleeding, fever, haematuria, syncope, dizziness, voiding difficulties/urinary retention and thromboembolism

- Later postoperative complications: pain in the hip/ thigh or the vagina, mesh extrusion, mesh erosion to the vagina or LUT, haematoma, abscess formation and nerve injury; in addition, new onset or worsening of any of the following: dyspareunia, vaginal discharge, voiding difficulties/urinary retention, long-term self-catheterisation, urgency/urgency incontinence.

Procedures for detecting, recording, evaluating and reporting AEs and SAEs

Detecting AEs and SAEs

All AEs and SAEs must be recorded from the time a participant consents to join the study until follow-up is complete.

Follow-up questionnaires will enquire about any AE/ SAE occurrence; in addition, participants will also be asked if they have been admitted to hospital and/or seen a healthcare professional.

\section{Recording AEs and SAEs}

Depending on severity, when an AE/SAE occurs, it is the responsibility of the investigator (or delegated medical personnel) to review appropriate documentation (eg, hospital notes, laboratory and diagnostic reports) related to the event. The investigator (or the delegated medical personnel) should then record all relevant information in the CRF and if required on the SAE form.

Information on SAE to be collected includes type and date of event, investigator assessment of severity and causality and any investigation/treatment required.

Planned hospital visits for conditions other than those associated with UI and/or its treatment will not be collected or reported. Further UI treatment will be recorded as a secondary outcome measure, but will not be reported as SAEs.

\section{Evaluating AEs and SAES}

All AEs will be assessed in respect of seriousness, relationship to study intervention, whether expected or unexpected, and therefore whether constituting an SAE by the local PI, chief investigator or their deputies.

\section{Assessment of seriousness}

The investigator should make an assessment of seriousness as defined in section 4.1.

\section{Assessment of causality}

The investigator must make an assessment of whether the $\mathrm{AE} / \mathrm{SAE}$ is likely to be related to any of the research procedures according to the following definitions:

- Related: resulted from any of the procedures required by the protocol, whether or not this procedure is the 
specific intervention under investigation, and whether or not it would have been administered outside the study as normal care

- Unrelated: where an event is not considered to be related to any of the research procedures.

\section{Assessment of severity}

The investigator should make an assessment of severity for each $\mathrm{AE} / \mathrm{SAE}$ and complete an SAE form should any of the SAE criteria in section 4.1 be met.

\section{Assessment of expectedness}

When assessing expectedness refer to the expected events (section 4.1).

The following is an example of the assessment of an AE: intraoperative bleeding will be collected as an $\mathrm{AE}$ on the operative form; however if $>500 \mathrm{~mL}$, an SAE form will be completed.

\section{Reporting AEs and SAEs}

Reporting responsibilities of the chief investigator

When an SAE form is uploaded onto the trial website, the trial manager and chief investigator will be automatically notified. The chief investigator or trial manager will notify the sponsor within 24 hours of receiving completed forms for 'un-expected' and 7 days of receiving completed forms for an 'expected' SAE. The sponsor will then provide the final assessment of the SAE.

The chief investigator (or trial manager) will report any 'related and unexpected SAEs' to the main Research Ethics Committee (REC) and the data monitoring committee (DMC) within 15 days of the chief investigator becoming aware of it. All other SAEs will be summarised and reported to the ethics committee, the funder, the DMC and the Trial Steering Committee (TSC) in their regular progress reports.

If all the required information is not available at the time of reporting, the investigator must ensure that any missing information is provided as soon as this becomes available. It should be indicated on the report that this information is follow-up information of a previously reported event.

\section{OUTCOME MEASURES}

This RCT assesses and compares the adjustable anchored SIMS versus tension-free SMUS with up to 3-year follow-up, in terms of patient-reported success rates, objective success rates, impact on urinary symptoms, complications, recovery, health-related QoL and sexual function, and costs to health services

\section{Primary outcome measure}

The primary outcome measure will be patient-reported success rate measured by the validated PGI-I at 12 months. Patient-reported success rates reflect patients' experience compared with the objective measures, which can overestimate the success of SUI surgery. ${ }^{27}$ The primary outcome is assessed by the PGI-I, a one-item questionnaire designed to assess the patient's impression of changes in her urinary symptoms. The PGI-I asks the patient to best describe her urinary symptoms, compared with how they were before the study intervention, on a 7-point scale scored as (1) 'very much better', (2) 'much better', (3) 'a little better', (4) 'no change', (5) 'a little worse', (6) 'much worse' or (7) 'very much worse'. 'Success' will be defined as responses of 'very much better' or 'much better'; this will determine whether the women are satisfied with their operation and hence consider their symptoms are resolved and not seek further treatments. The primary economic outcome will be incremental cost per QALY gained at 12 months. The above measures will also be assessed at 2 and 3 years.

\section{Secondary outcome measures}

The following are the secondary outcome measures:

- complications including LUT injuries, haemorrhage (blood loss $\geq 200 \mathrm{~mL}$ ), postoperative VD, pain, mesh extrusion/erosion, dyspareunia, long-term self-catheterisation, new-onset or worsening urgency/urgency incontinence, assessed as appropriate at 3 and 12 months, then yearly up to 3 years

- postoperative pain using a pain Numerical Rating Scale, assessed days 1-14

- objective success rates: assessed by 24-hour pad test at 12 months and yearly up to 3 years

- other LUT symptoms using the ICIQ-FLUTS long form and/or short form (ICIQ-SF) at 3 and 12 months and yearly up to 3 years

- health-related QoL profile (area under the curve) derived from EQ-5D, pain scores and ICIQ-LUTSqol measurements at 1,3 and 12 months and yearly up to 3 years

- impact on sexual function derived from ICIQ-FLUTSex/or PISQ-IR measurements at 12 months and yearly up to 3 years

- recurrence of SUI, reoperation rates for SUI, further treatment received such as physiotherapy, medical treatment (selective noradrenaline reuptake inhibitors and/or antimuscarinic treatment).

The secondary economic outcomes include the following:

- NHS and patient primary and secondary care resource use and costs at 12 months and yearly up to 3 years

- incremental cost-effectiveness derived from responses to the ICIQ-LUTS over the follow-up period at 12 months and yearly up to 3 years

- incremental net benefit (NB) calculated from the responses to the DCE at the end of the 3-year follow-up.

We will use the same assessment tools and QoL instruments used in our pilot RCT, which observed a 97\% response rate. 


\section{DATA COLLECTION AND PROCESSING}

\section{Measuring outcomes}

Participant follow-up questionnaires will be triggered by date of surgery up to 3 months, then by date of randomisation thereafter.

\section{Schedule of data collection}

The components of follow-up are shown in table 1 .

\section{Data processing}

Participants will be sent the follow-up questionnaires directly from the Study Office in Aberdeen and asked to return the completed questionnaires in prepaid envelopes. RNs will enter locally collected data in the centres. The Study Office will work closely with local (RNs) to ensure the data are complete and accurate. Extensive range and consistency checks will further enhance the quality of the data.

\section{SAMPLE SIZE, PROPOSED RECRUITMENT RATE AND MILESTONES}

\section{Sample size}

A non-inferiority design is appropriate for this trial because the proportion having success at 12 months in women managed with SMUS is high. Adjustable anchored SIMS is not hypothesised to increase this proportion, however may have other potential benefits as outlined previously. It is therefore important to show that SIMS is clinically non-inferior to SMUS and to measure these other dimensions (such as cost-effectiveness, mediated through shorter stay and earlier recovery, QoL mediated through less pain, and any safety signals via the complication rate) in an adequately powered, pragmatic RCT with long enough follow-up. It is essential therefore that the study is powered to demonstrate non-inferiority within an appropriate margin, and hence this clinical outcome is the correct choice as primary outcome. A $10 \%$ inferiority margin has been deemed by expert clinicians as the maximum inferiority margin in clinical effectiveness that would be accepted should SIMS prove to be superior in other outcomes such as shorter hospital stay, less postoperative pain, earlier recovery and more cost-effective. In such case, adjustable anchored SIMS would then reliably be able to be considered as a first-line surgical treatment of women with primary SUI.

Published literature suggests that $\mathrm{P} 1$, the percentage success rate at 12 months in the SMUS arm, will be about $85 \%$; identical results were confirmed by our pilot RCT. Estimating P2, the percentage of success in the adjustable anchored SIMS arm, is more difficult due to lack of published evidence; a crude meta-analysis of the 12-month outcome data from our multicentre pilot RCT and few other small studies indicates a similar P2 of $85 \%$.

Power estimates were explored by simulating trials of fixed sample size (using equal allocation) with binary responses generated by $\mathrm{P} 1=85 \%$ and $\mathrm{P} 2=85 \%$. Power was then estimated as the proportion of simulated trials where the lower bound of the two-sided CI satisfied P1-P2 $>-10 \%$. Simulations, run in Stata V.11.2 show that a trial of 275 per arm or 550 in total is required for the lower bound of the estimated $95 \%$ CI to rule inferiority at the specified level with $90 \%$ power. Adjustment for potential $15 \%$ dropout inflates the trial to 650 in total. For comparison, a trial of this size would have above $80 \%$ power to test superiority on secondary outcomes of difference in means of size one-quarter of an SD (or $90 \%$ power to detect an effect size of $0.28 \mathrm{SD}$ ).

In our multicentre pilot RCT, ${ }^{17} 131 / 137$ women $(95.6 \%)$ completed the 12-month follow-up and showed no significant differences in the patient-reported success rate (OR $0.895 ; 95 \%$ CI 0.344 to $2.330 ; \mathrm{p}=1.000$ ) between adjustable anchored SIMS (Ajust) and SMUS (TVT-O) groups. These results, together with similar results from other studies detailed above, ${ }^{16-21}$ provide assurances for the reliability of our sample size calculations. A statistical reviewer previously queried whether we had considered the implications if in fact the success of the two procedures were not identical but slightly different. If we consider success rates of $84 \%$ and $85 \%$, the study retains $90 \%$ power to detect a slightly larger margin of non-inferiority of around $11 \%$, and so to all intents and purposes a sample size of 650 remains adequate.

\section{Recruitment rates and milestones}

Our recruitment rate estimates are based on data from the pilot multicentre RCT comparing adjustable anchored SIMS (Ajust) with SMUS (TVT-O). We believe that these centres are representative of the UK; 137 women were recruited across six centres at a rate of 3.4 per centre per month. Overall, 137/181 (76\%) patients were willing to be randomised; however, we have used a more conservative estimate of $50 \%$ in our recruitment projection. Therefore, it has been estimated that in order to approach 1300 eligible patients to randomise the required 650 patients, 25 centres would need a throughput of at least 90 eligible patients per centre per year to recruit three patients per month. The recruitment projection is based on 18 months of recruitment (months 7-24 inclusive) and allows for set-up, holidays and waiting list times. We expect a staggered recruitment of centres with all centres active by the end of month 18. The first 45 patients will be recruited by month 12, 256 patients by month 18 and the remaining 367 patients by month 24, making a total of 668 patients.

Due to lower than predicted recruitment, a further extension has been granted to enable recruitment to reach at least 600 participants This increases the recruitment period so that the last participant will be recruited in month 44 . Please see the Gantt chart (figure 2) and recruitment projection (online supplementary appendix 4).

\section{ANALYSES PLAN}

\section{Statistical analysis}

Treatment groups will be described at baseline and follow-up using numbers (with percentages), means (with 


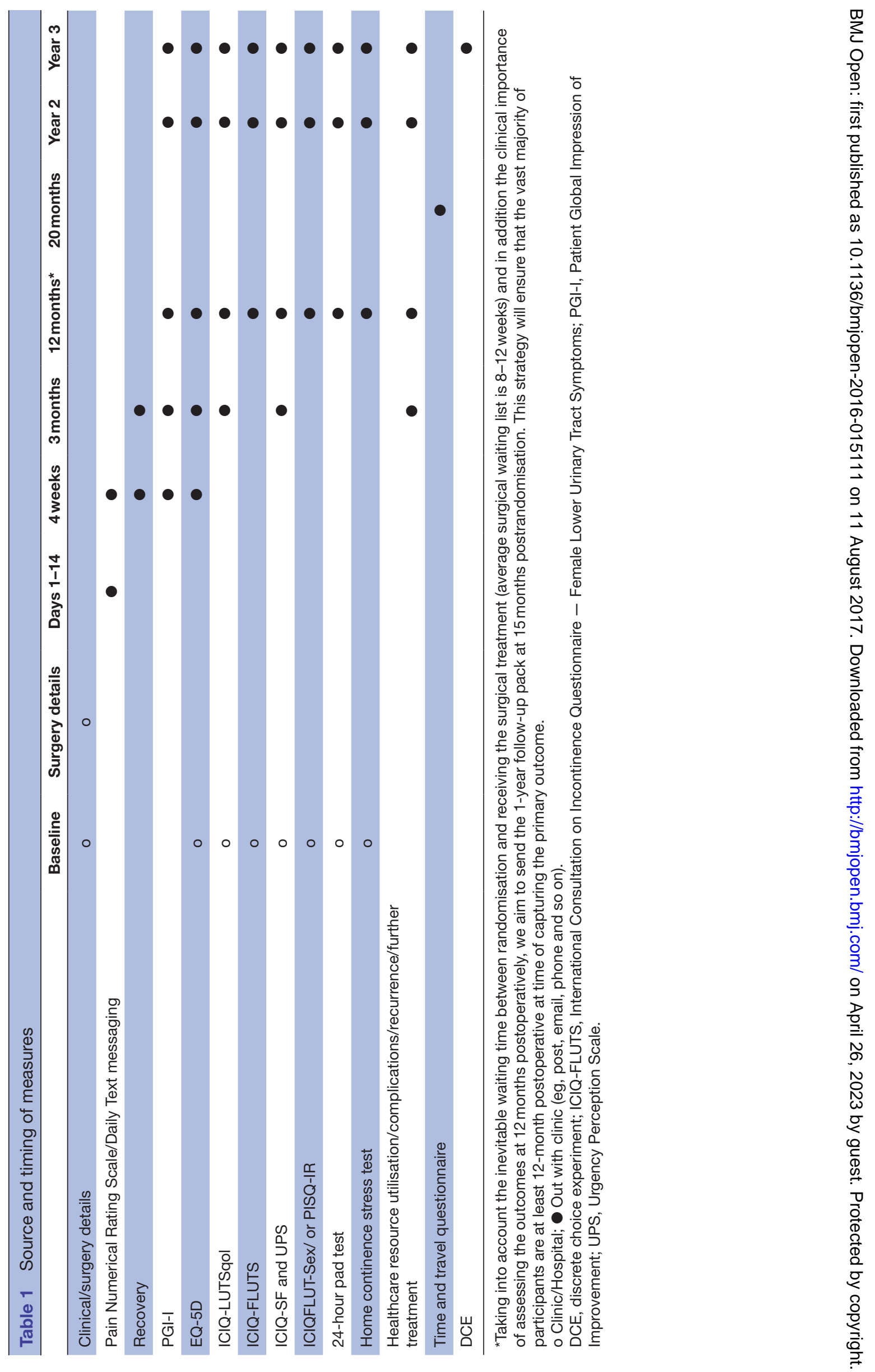


SD) and medians (with IQRs) where relevant. Primary and secondary outcomes will be compared using generalised linear models, with adjustment for design covariates. As standard we also adjust all our surgical RCTs for centre/ surgeon effects; adjustment for centre/surgeon will be by random effect in the trial analysis.

For the primary outcome, we plan to dichotomise the PGI-I responses with 'success' defined as 'very much better' or 'much better' and the rest of responses as failures; this will determine whether the women are satisfied with their operation and hence consider their symptoms are resolved and do not seek further treatments. In addition, this definition of 'success' is widely used within the research field of surgical treatment of SUI and was used in our pilot RCT, and therefore will allow comparing our results with other trials in the literature. We will also perform a secondary analysis using ordinal regression on the 7-point PGI-I scale, so potentially using more of the information in the outcome. However, we do not propose adopting this ordinal regression as the primary analysis since the underlying proportional odds model makes strong assumptions about the consistency of treatment effect across the levels of response, and particularly in the context of a non-inferiority design there may be departures from those assumptions that would interfere with establishing whether the simple hypothesis around the non-inferiority of the binary 'success' under the two operations had been shown.

The statistical analysis of the primary outcome will be by the usual intention-to-treat (ITT) and also a suitably defined per-protocol analysis (to reflect the unique nature of non-inferiority designs and the issue that ITT for such designs may not be the most conservative analysis and inflate the true type I error rate, given that in a non-inferiority design the null hypothesis is that the interventions are not non-inferior or equivalent). If the two approaches return material differences in interpretation, this will be investigated carefully. Results will be displayed as estimates and 95\% CIs derived from appropriate generalised linear models. CIs around observed differences will then be compared with the prespecified non-inferiority margin.

Secondary outcomes will be analysed similarly. Outcomes such as postoperative pain will be assessed under a superiority hypothesis as we believe that this will be lower in the intervention arm. As stated in the sample size section, there is above $80 \%$ power to detect a difference of a quarter of an SD under a superiority hypothesis.

All analyses will follow a carefully documented statistical analysis plan. Subgroup analyses (appropriately analysed by testing treatment by subgroup interaction) will explore possible treatment effect modification. The following are the prespecified subgroups:

- mixed incontinence versus pure stress incontinence

- urodynamic versus clinical diagnosis of SUI

- adjustable anchored SIMS versus each type of SMUS (ie, RP-TVT and TO-TVT separately)

- comparison of the main types of SIMS

- we will also include an exploratory subgroup analysis comparing those above and below the observed median age of the recruited women using a formal test of interaction

- responses to two validated sexual function questionnaire: ICIQ-FLUT-Sex versus PISQ-IR.

\section{Effect of pregnancy}

MUS procedures are generally offered to women after having completed their families, and therefore subsequent pregnancy is usually a rare event that is unlikely to be balanced between both trial arms. If a woman falls pregnant after receiving treatment within the RCT, her data will be censored at the time of confirmed pregnancy for the primary analysis. This small number of women will still be followed up for all outcome data as usual, and if the numbers warrant, a sensitivity analysis including them will be undertaken on the primary outcome.

The TSC and an independent Data Monitoring and Ethics Committee will be asked to review and comment on the statistical analysis plan prior to analysis. There are no plans for any formal interim analyses to be seen by the DMC. A single main analysis will be performed on the 12-month primary outcome and repeated on the 2-year and 3-year outcomes. The DMC will meet before recruitment begins, or as soon as practical, to agree the Terms of Reference and other procedures.

\section{ECONOMIC EVALUATION}

Our primary health economic evaluation will be from a health service provider's (NHS) perspective; however, we will also present data from a wider societal perspective.

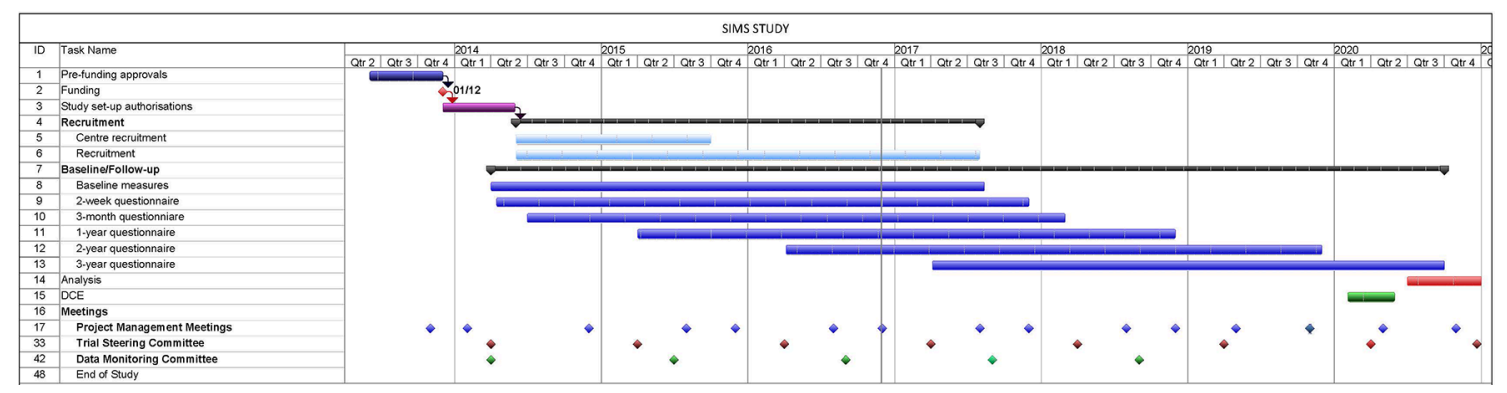

Figure 2 SIMS study Gantt chart. SIMS, Single-incision mini-slings. 
These data will include costs to patients of time and travel, costs to carers and family members, and costs to society as a whole, estimated from lost productivity as a result of time off work/away from normal activities.

\section{Collection of resource use and cost data}

Healthcare resource use will be collected using patient-administered questionnaires asking patients to retrospectively recall their contacts with healthcare professionals relating to their incontinence. This questionnaire will be administered at 3 and 12 months, then yearly for 3 years. It is generally accepted that patient recall is accurate up to 12 months, and it is highly unlikely that a patient would not remember significant events relating to their disease over this time period. Data collected will include secondary care contacts (hospital inpatient admissions, outpatient appointments) and primary care contacts (eg, GP contacts, nurse contacts, physiotherapist consultations) and prescription drug medications. These healthcare utilisation data will be combined with unit cost information for the use of specific resources using standard sources. ${ }^{29-31}$ Data on costs for each group (SIMS and SMUS) will be summed to provide an average cost per patient trial participant. Sensitivity analysis will be used to explore various distributions of cost data as well as various methods for the imputation of missing and censored data. We will provide a comprehensive range of deterministic sensitivity analyses to test any assumptions we make in our analysis on the overall results. For example, we will test best and worst case scenarios for the intervention cost (whether all procedures in the SIMS arm are conducted under GA or LA). The impact of any missing data and methods of data imputation on our results will also be tested. We will test the impact of these and a range of other sensitivity analyses to be determined as the trial progresses on all our results (eg, cost utility analysis and cost benefit analysis).

\section{Participant costs}

Out-of-pocket patient expenses (including the purchase of containment products), private healthcare costs, travel costs and costs associated with lost days at work will also be collected using the patient-administered questionnaire and incorporated into the patient perspective analysis. Costs of family members and/or carers will also be collected as part of the trial and reported.

\section{QUALITY OF LIFE}

Health state valuations will be based on the responses to the ICIQ-LUTSqol (baseline, 3, 12 months and annually over the follow-up period) and EQ-5D administered at baseline, 1, 3 and 12 months and annually over the follow-up period. These data will be transformed into utility values using standard algorithms. QALYs will be calculated, using the area under the curve methods, with any differences between groups being reported. Both measures will be compared and contrasted and tested for comparability in measuring outcomes for these women.

\section{COST-EFFECTIVENESS}

The analysis will use the estimates of mean costs and QALYs as described for each trial participant to estimate the incremental cost-effectiveness ratio at 12-month follow-up period and where appropriate the analysis will mirror that of the statistical analyses. Cost-effectiveness (cost per QALY gained) will also be reported over the 3-year follow-up period. The results of the analysis will be presented as incremental costs, effects and incremental cost per QALY. Bootstrapping of cost and QALY differences, as well as a range of one-way and multiway deterministic sensitivity analyses, will be conducted to address uncertainty in the estimates. Cost per QALY data will also be presented in the form of cost-effectiveness acceptability curves.

\section{DISCRETE CHOICE EXPERIMENT}

Previous research ${ }^{32}$ has suggested that EQ-5D questionnaire may not fully capture the benefits from successfully treating incontinence. They may not fully represent patient preferences for treatments and their associated outcomes. Therefore, we will conduct a DCE to elicit preference for the process, patient experience and health outcomes. A DCE presents respondents with a series of hypothetical choices that describe the choice alternatives by their underlying attributes; respondents are asked to choose the alternative they prefer. The values of the attributes vary across choice scenarios; by observing the peoples' choices, it is possible to infer their preferences over the attributes of each surgical procedure. The extent to which an individual values an intervention depends on the levels of these attributes. ${ }^{33}$ DCEs are commonly used to identify people's preferences in a variety of non-market situations/services/commodities. ${ }^{34-36}$

The attributes and levels for the DCE will be informed by systematic literature searching and advice sought from clinical experts. Attributes might include preferences for general/local anaesthetic, preferences for pain levels, cure and improvement rates, impact on activities of daily living, and so on. These attributes and levels will be combined to identify profiles that will be used to develop scenarios to present the study participants. The questionnaire will be piloted among a convenience sample to refine all practical aspects of the survey and to ensure that trade-offs can be made between the identified attributes. Once the pilot is complete and the questionnaire has been refined, it will be administered to the trial participants at the end of the 3-year follow-up.

Experimental design techniques will be used to generate an efficient set of choices from which preferences will be estimated. Logistic regression techniques will be used to analyse the response data. A cost attribute will be included so that willingness to pay (WTP) 
can be estimated. The results of the DCE information will be combined with the clinical outcomes estimated from the trial to provide an estimate of mean WTP for each of the two interventions. Results of the WTP aspect of the DCE will be presented as incremental NB between groups, where NB will be measured as WTP less the mean cost for each intervention. The intervention with the greatest NB will be deemed the most efficient. The results of this analysis will be compared and contrasted with the cost/QALY outcomes and will yield some information regarding the applicability of traditional QALY measurement to conducting economic evaluation in UI. The resultant costs and utilities will be used to estimate preference-based quality weights for this condition.

\section{ORGANISATION: TRIAL MANAGEMENT AND OVERSIGHT ARRANGEMENTS \\ Study Office in Aberdeen}

The SIMS trial is managed by the Study Office at the CHaRT based within the Health Services Research Unit (HSRU), University of Aberdeen. The staff in the Study Office, led by the trial manager, provides the day-to-day support for the clinical centres. The data coordinator organises all clerical aspects, including the postal questionnaires (mailing, tracking and entering returned data using the study web data entry portal). A quality assurance manager oversees and ensures the implementation CHaRT's standard operating procedures for trials, including observance of the appropriate principles of Good Clinical Practice.

At the centres, the RNs (or recruitment coordinators) are responsible for all local processes involved in identifying, consenting, randomising the participants, and ensuring the completion of the study baseline assessment and completion of the surgical intervention research records.

The SIMS Study Office team will meet formally at least monthly during the course of the study to ensure smooth running and troubleshooting.

\section{Local organisation in sites}

The local PI and RN will be responsible for all aspects of local organisation, including identifying, consenting and randomising the participants, along with facilitating the delivery of the intervention and notification of any problem or unexpected developments for the duration of the trial. They will be responsible for ensuring that study data are collected for baseline assessments, collecting and recording participant study data on study-specific case report forms and logging all the details onto the remote web-based data capture system as soon as practical after completion. The local PI will return all study documents to the Study Office in Aberdeen when requested.

\section{Project Management Group}

The study will be supervised by a Project Management Group (PMG). The chair of this group will be the chief investigator (Mohamed Abdel-Fattah) and will consist of representatives from the Study Office and grant holders. The PMG will meet every 3 months, including face to face in month 1 and month 6 in the first year. It is expected that, once the project is underway, the majority of these meetings will be held by teleconference; however, the PMG will also meet face to face at least annually. In addition, the PMG will also meet at the annual TSC meeting.

\section{Trial Steering Committee}

The study is overseen by a TSC. The membership of this committee comprised four independent members along with the chief investigator or a nominated delegate. The trial sponsors, other SIMS grant holders and key members of the central office (eg, the trial manager) can participate in TSC meetings but are not members. The funders will be notified in advance of meetings and a representative will be invited to attend. Other relevant experts may be invited to attend as appropriate. Details of the membership of the TSC can be found at the start of this protocol. CHaRT has adopted the TSC Charter adapted from the DAMOCLES Charter for DMCs and suggests to the independent TSC members that they adopt the Terms of Reference contained within. The TSC will meet approximately yearly.

\section{Data monitoring committee}

An independent DMC is made up of members listed at the start of this protocol, one of whom is an experienced statistician. The DMC will initially meet to agree its Terms of Reference. CHaRT has adopted the DAMOCLES Charter for DMCs and suggests to the independent DMC members that they adopt the Terms of Reference contained within.

The DMC meets regularly to monitor the study data, including unblinded trial data and any reported SAEs. If required, the DMC would make recommendations as to any modifications required to be made to the protocol or the termination of all or part of the trial.

\section{RESEARCH GOVERNANCE, DATA PROTECTION AND SPONSORSHIP \\ Research governance}

The trial will be run under the auspices of CHaRT based at HSRU, University of Aberdeen. This will ensure compliance with research governance and provide centralised trial administration, database support and economic and statistical analyses. CHaRT is a registered clinical trials unit with particular expertise in running multicentre RCTs of complex and surgical interventions.

The PMG will ensure through the TSC that adequate systems are in place for monitoring the quality of the study and appropriate expedited and routine reports, to a level appropriate to the risk assessment of the study. 


\section{Data protection}

All trial data will be kept strictly confidential and accessed only by the relevant members of the trial team. Participants' details will be stored on a secure database under the guidelines of the 1998 Data Protection Act, and regular checks and monitoring are in place to ensure compliance. Data are stored securely in accordance with the Act and archived to a secure data storage facility. The senior IT manager (in collaboration with the chief investigator) will manage access rights to the data set. Participants will be allocated an individual specific trial number and their details will be anonymised on the secure database. We anticipate that anonymised trial data may be shared with other researchers to enable international prospective meta-analyses. To comply with the 5th Principle of the Data Protection Act 1998, personal data will not be kept for longer than is required for the purpose for which it has been acquired.

\section{Sponsorship}

The University of Aberdeen and NHS Grampian are the cosponsors for the trial.

\section{QUALITY ASSURANCE}

The trial will be monitored to ensure that the study is being conducted as per protocol, adhering to research governance and the appropriate regulations. The approach to, and extent of, monitoring (specifying both central and on-site monitoring) will be specified in a trial monitoring plan, which is usually initially determined by a risk assessment, undertaken prior to start of trial.

\section{DATA HANDLING, RECORD KEEPING AND ARCHIVING}

Clinical data will be entered into the database by the local investigator and/or $\mathrm{RN}$ working in each hospital site, together with data from questionnaires completed at clinic. Questionnaires returned by post to the trial office will be entered there. Staff in the trial office will work closely with local RNs to ensure that the data are as complete and accurate as possible. Extensive range and consistency checks will further enhance the quality of the data.

The cosponsors are responsible for ensuring that trial data are archived appropriately. Essential data shall be retained for a period of at least 10 years following close of study.

\section{SATELLITE STUDIES}

It is recognised that the value of the study may be enhanced by smaller ancillary studies of specific aspects. Plans for these will be discussed in advance with the PMG. REC approval will be sought for any new proposal, if appropriate.

\section{ETHICS AND REGULATORY APPROVALS}

\section{Ethics approval}

The North of Scotland REC has reviewed and approved this study. The study will be conducted according to the principles of good clinical practice provided by the research governance guidelines. We believe this study does not pose any specific risks to individual participants beyond standard surgical procedures, nor does it raise any extraordinary ethical issues. Annual progress reports and a final report at the conclusion of the trial will be submitted to the North of Scotland REC within the timelines defined in the regulations.

\section{Finance and insurance}

The trial is funded by a grant awarded by the NIHR Health Technology Assessment programme. The necessary trial insurance is provided by the University of Aberdeen.

\section{Authorship and publication policy}

All RCTs conducted by CHaRT have a commitment to publish the findings of the research. At a minimum this trial will have a results paper published in a peer-reviewed medical/scientific journal. If all grant-holders and researcher staff fulfil authorship rules, group authorship will be used under the collective title of 'the SIMS Trial Group'. The chief investigator and possibly other members of the trial group will take responsibility for drafting the paper, and this will be recognised by line 'the CI (as primary author), followed by the other authors and the SIMS Trial Group'.

For reports that arise from the trial but where some members do not fulfil authorship rules (eg, specialist substudy publications), authorship should be attributed to chief investigator and the named individual(s) for the SIMS Trial Group.

To safeguard the integrity of the main trial, reports of explanatory or satellite studies will not be submitted for publication without prior arrangement from the PMG.

We intend to maintain interest in the study by publication of SIMS newsletters at intervals for staff and collaborators. Once the main report has been published, a lay summary of the findings will be sent in a final SIMS newsletter to all involved in the trial. Further details on the publication policy can be found in online supplementary appendix 5 .

\section{DISCUSSION}

The SIMS study is a key outcome study that should answer the important research question of whether adjustable SIMS should be used in clinical practice as a first-line surgical treatment option for women with primary SUI.

This study is a pragmatic, patient-oriented trial aiming to capture a true representation of the actual patient population. The inclusion/exclusion criteria were chosen to allow the capturing of the relevant patient group.

This trial seeks to follow standard pathways of care with the only additional intervention being randomisation 
between the two treatment strategies under test and collection of baseline and outcome information.

The results will inform clinicians and policy makers on the cost-effectiveness of this relatively new technology compared with the SMUS. The long-term follow-up in the SIMS study is crucial to address the long-term successes rate and AEs of MUS in general and SIMS in specific.

\section{Dissemination}

The dissemination plans include (1) Health Technology Assessment Program monograph, (2) presentation at international scientific meetings, (3) publications in high-impact, open-access, peer-reviewed journals, and (4) presentations at health economic and health services research meetings. The results of the trial will be included in the updates of NICE and the European Association of Urology guidelines; these two specific guidelines directly influence practice of all the UK and worldwide specialists, respectively. In addition, a plain English-language summary of the main findings and results will be presented for relevant patient organisations and communities, including the bladder and bowel foundation. This will ensure user relevance in dissemination of the results.

\section{Trial status}

The SIMS study is currently recruiting in $20 \mathrm{UK}$ research centres. The first patient was randomised on 4 February 2014, with follow-up to be completed at the end of February 2020.

\section{Author affiliations}

${ }^{1}$ Department of Obstetrics and Gynaecology, University of Aberdeen, Aberdeen, UK

${ }^{2}$ Health Services Research Unit (HSRU), University of Aberdeen, Aberdeen, UK

${ }^{3}$ Health Economics Research Unit, University of Aberdeen, Aberdeen, UK

${ }^{4}$ Spire Hospitals, Leeds, UK

${ }^{5}$ Centre for Healthcare Randomised Trials (CHRT), University of Aberdeen, Aberdeen, UK

${ }^{6} \mathrm{CHRT}$, University of Aberdeen, Aberdeen, UK

${ }^{7}$ Patient Representative, London, UK

${ }^{8}$ Chair in Surgery (Clinical), Academic Urology Unit, University of Aberdeen, Aberdeen, UK

Contributors MAF conceived the idea and wrote the protocol; JN contributed to the study design and the statistical analysis; GML contributed to the study design, the sample size calculation and wrote the statistical analysis plan; MK designed and wrote the economic analysis plan; RPA contributed to the clinical aspects of the protocol; JN contributed to the study design and clinical aspects of the protocol; JW contributed the layman summary as the patient representative; KM contributed to the study design, flow/Gantt charts; TD and AMD contributed to the trial oversight and the protocol amendments.

Funding This is a UK Collaborative Study funded by the NIHR Evaluation, Health Technology Assessment (HTA) Programme — Funder Number 12/127/157.

Competing interests MAF has been a previous speaker and or trainer for Bard, Astellas, Pfizer, AMS and Coloplast. He received travel grants to attend medical conferences from various companies and previously performed a research-led project funded by a research grant from Coloplast, which was received and administered by the University of Aberdeen. MAF was the Chairman of Scottish Pelvic Floor Network, which received support from different pharmaceutical and devices companies. MAF and RPA have provided industry-sponsorship proctorship training sessions to a number of surgeons training in SIMS prior to the study. None of the coapplicants have any financial interest and shares or sit on the advisory board for any relevant device companies.
Patient consent Detail has been removed from this case description/these case descriptions to ensure anonymity. The editors and reviewers have seen the detailed information available and are satisfied that the information backs up the case the authors are making.

Ethics approval North of Scotland Research Ethics Committee.

Provenance and peer review Not commissioned; externally peer reviewed.

Open Access This is an Open Access article distributed in accordance with the Creative Commons Attribution Non Commercial (CC BY-NC 4.0) license, which permits others to distribute, remix, adapt, build upon this work non-commercially, and license their derivative works on different terms, provided the original work is properly cited and the use is non-commercial. See: http://creativecommons.org/ licenses/by-nc/4.0/

(C) Article author(s) (or their employer(s) unless otherwise stated in the text of the article) 2017. All rights reserved. No commercial use is permitted unless otherwise expressly granted.

\section{REFERENCES}

1. Urinary incontinence; full guidline. CG40 [document on the internet]. London: National Insititute for Health and Clinical Excellence 2006 http://guidance.nice.org.uk/CG40/Guidance/pdf/English (accessed January 2013).

2. Perry S, Shaw C, Assassa P, et al. An epidemiological study to establish the prevalence of urinary symptoms and felt need in the community: the Leicestershire MRC Incontinence Study. Leicestershire MRC Incontinence Study Team. J Public Health Med 2000;22:427-34.

3. Fultz N, Girts T, Kinchen K, et al. Prevalence, management and impact of urinary incontinence in the workplace. Occup Med 2005;55:552-7.

4. Turner DA, Shaw C, McGrother CW, et al. The cost of clinically significant urinary storage symptoms for community dwelling adults in the UK. BJU Int 2004;93:1246-52.

5. Fultz NH, Burgio K, Diokno AC, et al. Burden of stress urinary incontinence for community-dwelling women. Am J Obstet Gynecol 2003;189:1275-82.

6. Papanicolaou S, Pons ME, Hampel C, et al. Medical resource utilisation and cost of care for women seeking treatment for urinary incontinence in an outpatient setting. examples from three countries participating in the PURE study. Maturitas 2005;52.

7. Abrams P, Artibani W, Cardozo L, et al. Clinical manual of incontinence in women. Health Publications Ltd 2005.

8. Jacklin P, Duckett J, Renganathan A. Analytic model comparing the cost utility of TVT versus duloxetine in women with urinary stress incontinence. Int Urogynecol J 2010;21:977-84.

9. Ogah J, Cody JD, Rogerson L. Minimally invasive synthetic suburethral sling operations for stress urinary incontinence in women. Cochrane Database Syst Rev 2009:CD006375. Art. No.: CD006375. DOI.

10. Assassa $P$, Moran $P$, Duckett $J$, et al. Stress incontinence surgery in the UK (1). Pre-operative work up and intra-operative complications. analysis of the british society of urogynaecology database. Neurourol Urodyn 2010;29:809-10.

11. Lucas MG, Bosch RJ, Burkhard FC, et al. EAU guidelines on surgical treatment of urinary incontinence. Eur Urol 2012;62:1118-29.

12. Giberti C, Gallo F, Cortese P, et al. The suburethral tension adjustable sling (REMEEX system) in the treatment of female urinary incontinence due to 'true' intrinsic sphincter deficiency: results after 5 years of mean follow-up. BJU Int 2011;108:1140-4.

13. Barrington J, Archer R, Kulkarni M, et al. The TRT Female Remeex System ${ }^{\circledR}$ for Recurrent Female stress Urinary incontinence: a 5-year follow-up study. J Obstet Gynaecol 2013;33:391-3.

14. Gadjiev N, Tabaza R, Kirschner-Hermanns R. Mini-sling: what is known about anchorage systems? Neurourol Urodyn 2012;31:709-1102.

15. Kocjancic E, Sedlar A. A strength comparison of immediate and delayed extraction forces of 5 different single incision slings anchor types: an animal model. Int Urogynaecol J (Suppl 2012;2:S115.

16. Abdel-Fattah M, Agur W, Abdel-All M, et al. Prospective multicentre study of adjustable single-incision mini-sling (Ajust(®)) in the management of stress urinary incontinence in women: 1-year followup study. BJU Int 2012;109:880-6.

17. Mostafa A, Agur W, Abdel-All M, et al. Multicenter prospective randomized study of single-incision mini-sling vs tension-free vaginal tape-obturator in management of female stress urinary incontinence: a minimum of 1-year follow-up. Urology 2013;82:552-9. 
18. Schweitzer KJ, Cromheecke AK, Milani HW, et al. A Randomized Controlled Trial comparing the Tvt- $\mathrm{R}$ with the Ajust $\AA$ as Primary Surgical treatment of Female stress Urinary incontinence. Int Urogynecol J 2012;23(Suppl 2):S77-S78.

19. Cornu JN, Ciofu C, Sebe P, et al. Cure of women stress urinary incontinence with the Ajust single Incision Sling: one year results. Neurourol Urodyn 2011;30:868.

20. Lucente V, Cornu JN, Sebe P, et al. Ajust single incision transobturator sling procedure for stress urinary incontinenvce in women. Int Urogynecol J 2011;22(Suppl 2):S1041.

21. Meschia M, Barbacini $P$, Baccichet $R$, et al. Short-term outcomes with the Ajust ${ }^{\mathrm{TM}}$ system: a new single incision sling for the treatment of stress urinary incontinence. Int Urogynecol J 2011;22:177-82.

22. Mostafa A, Lim CP, Hopper L, Madhurvata P, et al. Singleincision mini-slings versus standard midurethral slings in surgical management of female stress urinary incontinence: an updated systematic review and meta-analysis of effectiveness and complications. Eur Urol 2014;65.

23. Sivaslioglu AA, Unlubilgin E, Aydogmus S, et al. A prospective randomized controlled trial of the transobturator tape and tissue fixation mini-sling in patients with stress urinary incontinence: 5-year results. J Urol 2012;188:194-9.

24. Naumann G, Hagemeier T, Zachmann S, et al. AjustTM fully adjustable single incision sling for the treatment of stress urinary incontinence: 1 year follow-up on a new minimal-invasive treatment for female SUI. Int Urogyneco J 2011;22(Suppl 2):S1050.

25. Boyers D, Kilonzo M, Mostafa A, et al. Single incision mini-slings versus standard mid-urethral slings in surgical management of female stress urinary incontinence: a costeffectiveness analysis alongside a randomised controlled trial. Neurourol Urodyn 2012;31:726-7.

26. Ulmsten U, Henriksson L, Johnson P, et al. An ambulatory surgical procedure under local anesthesia for treatment of female urinary incontinence. Int Urogynecol J Pelvic Floor Dysfunct 1996;7:81-6.
27. Delorme E. [Transobturator urethral suspension: mini-invasive procedure in the treatment of stress urinary incontinence in women]. Prog Urol 2001;11:1306-13.

28. de Leval J. Novel surgical technique for the treatment of female stress urinary incontinence: transobturator vaginal tape inside-out. Eur Urol 2003;44:724-30.

29. NHS speciality costs: acute costs database on the internet. Edinburgh: Information Services Division (ISD) Scotland 2010 http:// www.isdscotland.org/Health-Topics/Finance/Costbook/SpecialityCosts/Acute-Surgical.asp (accessed Jan 2013).

30. NHS reference costs 2011-12 document on the internet. UK Department of Health 2012http://www.dh.gov.uk/health/2012/11/ 2011-12-reference-costs/ (accessed Jan 2013).

31. British National Formulary website on the internet. British Medical Association and Royal Pharmaceutical Society. 2013 http://www.bnf. org/bnf/index.htm (accessed Jan 2013).

32. Imamura M, Abrams P, Bain C, et al. Systematic review and economic modelling of the effectiveness and cost-effectiveness of non-surgical treatments for women with stress urinary incontinence. Health Technol Assess 2010;14:1-188, iii-iv.

33. Ryan M, Watson V, Amaya-Amaya M. Methodological issues in the monetary valuation of benefits in healthcare. Expert Rev Pharmacoecon Outcomes Res 2003:3:717-27.

34. Bateman I, Carson R, Day B, et al. Economic valuation with stated preference techniques: a manual: UK Department of Transport; Edward Elgar Publishing, 2002.

35. Hensher DA, Rose J, Greene WH. The implications on willingness to pay of respondents ignoring specific attributes. Transportation 2005;32:203-22.

36. Louviere JJ, Hensher DA, Swait J. Stated choice methods: analysis and application. Cambrideg: Cambridge University Press, 2000. 